\title{
La empleabilidad de los jóvenes en España: Explicando el elevado desempleo juvenil durante la recesión económica
}

\author{
The employability of young people in Spain: Explaining the high youth unemployment \\ during the economic downturn
}

\section{Almudena Moreno Minguez ${ }^{1}$}

Resumen: Los datos presentados en este artículo ponen de manifiesto que el elevado desempleo de los jóvenes en España es un hecho estructural que se explica fundamentalmente por la destrucción de empleos temporales, las características del sistema productivo y por los desajustes existentes entre la formación y el mercado laboral. Tiene por objetivo analizar las causas del elevado desempleo juvenil en España y las circunstancias que condicionan la empleabilidad. La metodología utilizada se ha basado en la exploración de datos secundarios procedentes de las Encuestas de Juventud, Eurostat y la Encuesta de Población Activa. El artículo subraya que la tasa de desempleo no se distribuye homogéneamente entre todos los jóvenes, afectando fundamentalmente a los más jóvenes, lo menos formados y los pertenecientes a las clases bajas y de otra nacionalidad. Estas circunstancias se evidencian, por un lado, en el elevado desempleo de los jóvenes menores de 25 años, el elevado fracaso escolar y, por otro lado, en la sobre-cualificación de los jóvenes.

Palabras clave: Formación, jóvenes, empleabilidad, abandono escolar temprano, desempleo.

\begin{abstract}
The data presented in this article evidence that the high youth unemployment in Spain is a structural fact which is explained by the destruction of temporary jobs, production system characteristics and the mismatches between education and labour market. Analyze the causes of high youth unemployment in Spain and the circumstances that affect employability. The methodology is based on the exploration of secondary data from the Youth Surveys, Eurostat and the Labour Force Survey. The article highlights the uneven incidence of unemployment among young people. Unemployment is concentrated primarily among younger, less educated and those belonging to the lower classes and other nationality. These results show a high unemployment among young people under 25 years, the high level of school dropouts and the high incidence of over-education of the young. The methodology is based on the exploration of secondary data from the Youth Surveys, Eurostat and the Labour Force Survey.

Keywords: Education, youth employability, early school leaving, unemployment.
\end{abstract}

\footnotetext{
${ }_{1}$ Universidad de Valladolid. Departamento de Sociología y Trabajo Social. Campus Maria Zambrano. España. Correspondencia: Plaza Alto de los Leones, 1, 40005 Segovia, España. E-mail: almudena@soc.uva.es "El manuscrito es el resultado de una investigación sobre la situación de los jóvenes españoles financiada por el Instituto de la Juventud de España, Proyecto I+D+I 2011-2012".

Recibido: 21/07/2014; Aceptado: 12/03/2015.

http://dx.doi.org/10.18004/riics.2015.julio.3-20 


\section{INTRODUCCIÓN}

En el actual contexto de crisis económica el concepto de "empleabilidad" se ha instalado en el discurso económico y político erigiéndose como un factor clave para activar y fomentar el empleo en una etapa fundamental del proceso de transición a la vida adulta (Shanahan, 2000; Moreno Minguez, 2012). Desde la perspectiva económica se entiende como tal un conjunto de factores, fundamentalmente relacionados con la formación, que satisfacen las necesidades de los empleadores respecto a la cualificación de sus trabajadores. Es un concepto de dificil delimitación debido a la extensión de sus componentes y a lo cambiante que es en la actualidad el mercado laboral debido, entre otras razones, a los constantes avances tecnológicos y a las turbulencias financieras.

Generalmente este término se asocia con el capital humano que incorpora el individuo como trabajador a la empresa para generar valor añadido y por tanto satisfacer las exigencias de productividad del mercado laboral. Este concepto generalmente se utiliza para valorar y analizar las situaciones formativas y profesionales que han generado la situación de empleo/desempleo. Desde hace una década este término es asumido en la literatura científica y por los organismos internacionales como expresión de las relaciones entre calificaciones recibidas por la educación y el proceso de inserción laboral y se presenta como el marco referencial para la estrategia Europa 2020 (European Union, 2013; OECD, 2013a).

El incremento del desempleo juvenil, consecuencia de la crisis económica, con especial intensidad en España, ha intensificado el debate sobre las causas que lo explican y cómo se asocia con el concepto de empleabilidad de este colectivo (Banco de España, 2013; Blázquez, 2005; Dolado, Jansen, Felgueroso, Fuentes y Wölfl, 2013). Los investigadores parecen estar de acuerdo en que las decisiones personales respecto a formarse, continuar con los estudios o abandonarlos están influenciados no sólo por factores de tipo individual motivacional sino también por factores familiares, de clase social, por la situación del mercado laboral y las propias condiciones macroeconómicas (Breen y Goldthorpe, 1999; Erikson y Jonsson, 1996; Schoon, 2008).

En relación con esta temática contamos con una amplia bibliografia acerca de cómo las competencias, las capacidades, las motivaciones personales, la familia, la escuela y la estructura del mercado laboral son factores explicativos claves de las trayectorias laborales de los jóvenes, pero son escasos los estudios y análisis empíricos que hayan resumido todos estos factores en un índice relativo de empleabilidad de los jóvenes con el fin de analizar los desajustes existentes entre la formación recibida por los mismos y la fallida inserción en el mercado laboral 
(Rodríguez Cuba, 2009; OIT, 2013; Dolado, Felgueroso y Jimeno, 2000). Teniendo en cuenta las limitaciones existentes en torno a este concepto, la propuesta de análisis que aquí se presenta tiene como objetivo analizar de forma explorativa y descriptiva la empleabilidad de los jóvenes en España a través del análisis de las diferentes situaciones individuales (formativas, clase social, sexo y nacionalidad) de estos jóvenes en un contexto productivo decadente e institucional de limitado estímulo público a la creación de empleo como consecuencia del recorte en gasto público provocado por la necesidad de reducir el déficit público.

Los debates existentes en la literatura científica sobre las causas del elevado desempleo juvenil en España son numerosos y en determinados casos contradictorios. Así nos encontramos con una línea de investigación que explica la reducida empleabilidad de los jóvenes por sus carencias formativas y el elevado abandono escolar temprano, factores que en momentos de recesión económica han acentuado las dificultades para emplear a determinados colectivos de jóvenes con reducida cualificación y formación. De hecho hay un acuerdo generalizado sobre el hecho de que los problemas fundamentales del mercado de trabajo español y sobre todo de los jóvenes se reducirian significativamente con una mejora del nivel y la calidad formativa de los trabajadores (Cabrales, Dolado y Mora, 2013; Ramón García, 2011). En este debate los mal llamados "ninis" (ni estudian ni trabajan) han sido utilizados para justificar el alarmante crecimiento del desempleo juvenil en España. Sin embargo numerosas investigaciones han desmontado la incidencia de este fenómeno, ya que no llegan al $2 \%$ y la mayoría de los casos se trata de jóvenes formados que no encuentran acople en el mercado laboral (Instituto de la Juventud, 2011; Moreno Mínguez, 2013).

En el otro extremo se encuentran los investigadores que subordinan el elevado desempleo juvenil a las fallas del sistema productivo español, anclado durante años en la inflación del sector de la construcción, que ha dejado sin oportunidades laborales a muchos jóvenes cuando irrumpe la crisis económica, convirtiendo el desempleo juvenil en un fenómeno estructural del sistema productivo (Felgueroso, 2012). Según los análisis realizados por Garrido (2012) contamos con suficientes evidencias empíricas para avalar que el problema del desempleo no es resoluble únicamente en términos exclusivos de formación. De hecho Pissarides (2011) ha documentado cómo la diferencia clave entre el crecimiento del empleo en EE.UU y su estancamiento en Europa se debe a la expansión en América de los puestos de atención al público (comercio y servicios directos), salud (sanidad y cuidados a las personas mayores y a los niños) y educación (sobre todo en las fases iniciales). La amplia dedicación de los inmigrantes en España a estas tareas, generalmente en la economía sumergida y el 
mantenimiento de la inmigración a pesar de la crisis evidencian que las políticas laborales han dificultado la extensión de empleabilidad en estos sectores para los españoles, ya que los empleos de menor nivel son los que tienen la cotización a la seguridad social más alta (Garrido, 2012), por lo que la argumentación de Pissarides podría ser extrapolada al caso español para explicar el elevado desempleo juvenil.

En definitiva, los investigadores que hablan de los déficit formativos se refieren al elevado desempleo de los jóvenes no cualificados en comparación con los jóvenes formados y los que achacan este fenómeno al sistema productivo se refieren a la limitada capacidad de absorción del sistema productivo español de los jóvenes talentos cualificados, lo que se refleja en la sobrecualificación y la emigración a otros países europeos de los jóvenes mejor formados (Moreno Mínguez, 2013).

Desde una perspectiva institucional hay estudios que apuntan a las politicas laborales como otro factor interviniente clave en la explicación de esta situación. Son varios los trabajos que dan cuenta de la rigidez del mercado laboral y la segmentación del mismo provocados por las políticas laborales en España (Council of the European Unión, 2010; Guell y Petrongolo, 2007; González y Miles, 2012). En el contexto europeo desde hace unos años hay un interés creciente por diseñar un conjunto de directrices politicas que sirvan como marco de referencia a los Estados nacionales para favorecer el empleo juvenil (Council of the European Union, 2010; European Commission, 2012; European Commission, 2013).

Si bien no hay acuerdo sobre las causas de la reducida empleabilidad de los jóvenes españoles, sí que hay cierto consenso en torno a que el desempleo no afecta por igual a todos los jóvenes, puesto que determinados colectivos como los de menor edad, los menos formados, los pertenecientes a las clases sociales más humildes y a otras nacionales o minorias étnicas son los más afectados por la destrucción de empleo. El sexo es otra variable interviniente, puesto que las mujeres jóvenes parecen estar resistiendo mejor que los hombres al desempleo, no sabemos si por una reacción ante el empeoramiento de la situación laboral de sus compañeros o como el resultante de la rentabilización que ha supuesto su capitalización en educación en décadas pasadas (Moreno Mínguez, 2013).

Por lo tanto, los estudios realizados a este respecto evidencian que los procesos estructurales e institucionales generados en torno a la crisis económica generan más heterogeneidad que homogeneidad en las trayectorias laborales y formativas de los jóvenes (Beck y Beck-Germsheim, 2002; Schoon y Silbereisen, 2009). Otra consecuencia que parece derivarse de la actual crisis económica es que 
el futuro de los mercados laborales podrian acentuar la polarización de las transiciones laborales de los jóvenes puesto que la cambiante naturaleza de los requerimientos competenciales de los puestos de trabajo de alta cualificación y la mayor demanda de perfiles semi-cualificados o de baja cualificación puede acrecentar la desigualdad laboral entre jóvenes, así como acrecentar el desempleo en determinados colectivos de jóvenes.

\section{METODOLOGÍA}

En base a estas premisas teóricas expuestas en el epígrafe anterior en este artículo se presenta un análisis exploratorio partiendo de la hipótesis de que el desempleo juvenil se explica en función de múltiples variables, además de no afectar por igual a todos los jóvenes en España, siendo la posición ocupada en la estructura social, la formación y la nacionalidad variables explicativas claves para explicar esa diversidad. A estos determinantes estructurales de orden individual se unen otros factores de indole institucional referidos a las politicas laborales y al propio mercado laboral, característicos de la sociedad española, que explicarian la acusada incidencia del desempleo en determinados colectivos de jóvenes en España.

La metodología utilizada para llevar a cabo este análisis descriptivo se ha basado en la explotación de los datos procedentes de las Encuestas de Juventud realizadas por el Instituto de la Juventud de España en los años 2008 y 2012, así como en la explotación de los datos procedentes de la Encuesta de Población Activa desde el año 2000 y Eurostat para los datos comparados. Nuestra variable dependiente ha sido el desempleo y ocupación de los jóvenes, además de utilizar otras variables independientes tales como la edad, el sexo, la clase social, la nacionalidad y la formación, con fines estrictamente descriptivos. El objetivo final de este proceder metodológico es que este análisis exploratorio nos permita elaborar algún tipo de modelo explicativo sobre la reducida empleabilidad de los jóvenes y por tanto el elevado desempleo juvenil en España.

\section{RESULTADOS Y DISCUSIÓN}

Dos de las características más destacadas de la economía española desde mediados de los noventa han sido por un lado la fuerte segmentación del mercado laboral causada por una legislación de protección al empleo que ha venido incentivando el uso masivo de la contratación temporal, especialmente a través de los contratos de duración determinada, y por otro lado, la fuerte reducción de la 
tasa de crecimiento de la productividad total de los factores, variable que refleja el limitado avance tecnológico y de la inversión en $\mathrm{I}+\mathrm{D}+\mathrm{I}$, así como el reducido nivel de la formación de empresarios y trabajadores (Dolado, García y Jimeno, 2002; Cabrales et al, 2013). A esto se une que uno de los efectos más destacados de la crisis económica en España ha sido la destrucción de empleo temporal y la reducción del gasto público en $\mathrm{I}+\mathrm{D}+\mathrm{I}$ y formación, lo que ha penalizado especialmente al empleo de los jóvenes.

Como consecuencia de todo esto y haciendo una lectura en términos agregados, el desempleo juvenil ha aumentado considerablemente en España desde el inicio de la crisis, habiendo manteniendo históricamente un ratio más elevado que la media europea. La recesión económica ha tenido un impacto considerable en empleo juvenil. La evolución del desempleo por grupos de edad evidencia que el grupo de edad más castigado ha sido el de los menores de 25 años (Figura 1). Una tendencia similar pueden observarse cuando se compara la evolución que ha tenido el desempleo de los "jóvenes adultos" (25 a 29 años), si bien con unos valores significativamente inferiores a los del grupo de menor edad. El empleo juvenil de 16 a 29 años ha registrado en España entre 2008 y 2012 una pérdida de casi dos millones de personas, lo que representa un $67 \%$ de la población que ha perdido su trabajo en ese periodo (Rocha, 2012).

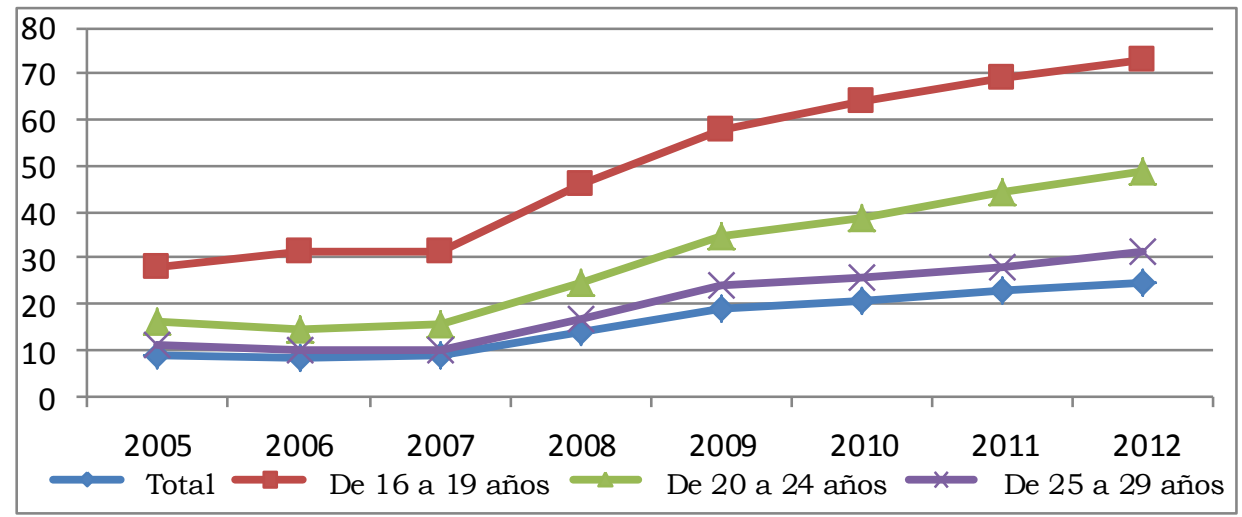

Figura 1. Tasas de paro por grupos de edad, España, 2005-2012.

Fuente: Elaboración propia a partir de la EPA, varios años, INE. 
Estos datos apuntan, según los investigadores, a cierta disfuncionalidad del sistema productivo español (Garrido, 2012; Felgueroso, Hidalgo y Jiménez-Martín, 2010, Bentolilla, Cahuc, Dolado y Le Barbanchon, 2012). Las evidencias empíricas que avalan esta tesis nos remiten por un lado a las características de los empleos que se han creado y destruido en el colectivo de los jóvenes desde el inicio de la crisis y, por otro lado, a la evolución que ha tenido la sobrecualificación. Con respecto al primer indicador destaca el hecho de que se ha perdido empleo entre los jóvenes en todas las ramas de actividad pero especialmente entre los directivos/gerentes, entre los empleados administrativos y las ocupaciones elementales, es decir, fundamentalmente en los dos extremos de la pirámide ocupacional (Moreno Mínguez, 2008; 2013).

Tal y como se puede apreciar en la tabla 1, destaca la elevada concentración sectorial de los jóvenes en el año 2008 en sectores que han sido muy afectados por la crisis como la construcción, la industria manufacturera y el comercio. Por lo tanto, los factores que explican la vulnerabilidad del empleo juvenil están relacionados en parte con las características del sistema productivo español que empleó a los jóvenes en la etapa expansiva.

Tabla 1. Ocupados jóvenes por grupo de edad, sexo y rama de actividad, (miles).

\begin{tabular}{|c|c|c|c|c|c|c|c|c|}
\hline \multirow{3}{*}{$\begin{array}{l}\text { Ocupados por grupo de edad, sexo y rama de actividad } \\
\text { Unidades:Miles de personas }\end{array}$} & & & \multicolumn{6}{|c|}{ Grupo de edades } \\
\hline & \multicolumn{2}{|c|}{ 16-29 años } & \multicolumn{2}{|c|}{$16-19$ años } & \multicolumn{2}{|c|}{ 20-24 años } & \multicolumn{2}{|c|}{ 25-29 años } \\
\hline & 2012 & 2008 & 2012 & 2008 & 2012 & 2008 & 2012 & 2008 \\
\hline Agricultura, ganaderia, silvicultura y pesca & 93,6 & 131 & 6,5 & 16,2 & 29,9 & 42,2 & 57,2 & 72,6 \\
\hline Industrias extractivas & 4,5 & 13,3 &.. & 0,3 & 1,7 & 4,4 & 2,8 & 8,6 \\
\hline Industria manufacturera & 290,5 & 655,8 & 5,8 & 46,6 & 78,7 & 220,7 & 206 & 388,5 \\
\hline Suministro de energía eléctrica, gas, vapor y aire acondicionado & 15,4 & 12,9 & 0,9 & 0,6 & 4,2 & 2,7 & 10,3 & 9,6 \\
\hline $\begin{array}{l}\text { Suministro de agua, actividades de saneamiento, gestión de residuos y } \\
\text { descontaminación }\end{array}$ & 14,7 & 20,6 & 0,3 & 1,9 & 4 & 4 & 10,4 & 14,7 \\
\hline Construcción & 159 & 657,9 & 4,9 & 51,8 & 48,1 & 219,1 & 106 & 387 \\
\hline $\begin{array}{l}\text { Comercio al por mayor y al por menor; reparación de vehículos de } \\
\text { motor y motocicletas }\end{array}$ & 523,9 & 874,7 & 19,8 & 79,4 & 171,7 & 319,3 & 332,4 & 476 \\
\hline Transporte y almacenamiento & 84,5 & 160,9 & 3,2 & 9,2 & 19,5 & 47,2 & 61,8 & 104,5 \\
\hline Hosteleria & 322,2 & 429,1 & 19,4 & 43,2 & 113,1 & 169,4 & 189,7 & 216,5 \\
\hline Información y comunicaciones & 102,7 & 157,3 & 0,9 & 4,8 & 23,9 & 46,4 & 77,9 & 106,1 \\
\hline Actividades financieras y de seguros & 39,7 & 101,1 & .. & 0,8 & 6,8 & 23 & 32,9 & 77,3 \\
\hline Actividades inmobiliarias & 9,4 & 14,9 & .. & 0,5 & 3,2 & 2,8 & 6,2 & 11,6 \\
\hline Actividades profesionales, cientificas y técnicas & 137,1 & 213 & 1,1 & 6,5 & 20,7 & 47 & 115,3 & 159,5 \\
\hline Actividades administrativas y servicios auxiliares & 107,3 & 172,8 & 3,8 & 9,8 & 30,5 & 58,7 & 73 & 104,3 \\
\hline Administración Pública y defensa; Seguridad social obligatoria & 107,4 & 152 & 1,2 & 8,2 & 25,6 & 42 & 80,6 & 101,8 \\
\hline Educación & 166,5 & 179,3 & 3,4 & 7 & 47 & 44,7 & 116,1 & 127,6 \\
\hline Actividades sanitarias y de servicios sociales & 188,9 & 232,4 & 1,9 & 6,1 & 47,3 & 71,6 & 139,7 & 154,7 \\
\hline Actividades artísticas, recreativas y de entretenimiento & 76,8 & 98 & 6,2 & 11,7 & 31,4 & 40,8 & 39,2 & 45,5 \\
\hline Otros servicios & 81,2 & 126,3 & 4 & 8,2 & 22,8 & 48,7 & 54,4 & 69,4 \\
\hline $\begin{array}{l}\text { Actividades de los hogares como empleadores de personal doméstico y } \\
\text { como productores de bienes y servicios para uso propio }\end{array}$ & 93,5 & 165,9 & 2,6 & 5,5 & 23,8 & 55,5 & 67,1 & 104,9 \\
\hline Actividades de organizaciones y organismos extraterritoriales & 0,9 & 0,5 &.. & .. &.. & 0,3 & 0,9 & 0,2 \\
\hline
\end{tabular}

Fuente: Elaboración propia a partir de la EPA, INE, Informes de Juventud, 2013. 
La destrucción de empleo en España durante la crisis ha sido generalizada, pero no ha afectado a todos los tipos de empleo de la misma forma (Bancaja-IVIE, 2011). Cuando se considera la evolución por ocupaciones se observa que los puestos de trabajo más cualificados no han caído sino que se han mantenido (impulsando el empleo total un 0,1\%) (Tabla 2). En España el ajuste se ha concentrado fundamentalmente en las ocupaciones propias de los trabajadores menos cualificados, equivaliendo a un descenso del 2,25\% del empleo total. Esa evolución es más negativa que la del conjunto de la UE y peor que en el resto de las economías europeas. En Francia y en Italia ese tipo de puestos de trabajo incluso ha crecido. El resto de ocupaciones de cualificación intermedia son las más numerosas y también las que concentran el grueso de la reducción de empleo en España, suponiendo una caída que equivale al 7,3\% del empleo total.

Tabla 2. Trabajadores en ocupaciones de alta cualificación (\%).

\begin{tabular}{lcc} 
& $\mathbf{2 0 0 7}$ & $\mathbf{2 0 1 1}$ \\
\hline España & 31,8 & 35,2 \\
Reino Unido & 42,1 & 43,7 \\
Francia & 40,3 & 42,4 \\
Italia & 40,7 & 38,3 \\
\hline
\end{tabular}

Fuente: Banca Caja- Ivie, 2012, Informe de Juventud, 2013.

Otro indicador que apunta a las deficiencias del sistema productivo es la incapacidad relativa que tiene de absorber a los jóvenes cualificados (Martínez, 2013; Pérez y Hernández, 2013). En los datos comparados ofrecidos por Eurostat presentados en la tabla 3, se puede observar que España tiene la mayor proporción de jóvenes sobrecualificados (32,5\%) seguida de Reino Unido $(25 \%)$ y Francia $(20,7 \%)$ y muy por encima de la media de la Europa de los $27(21,2 \%)$. Si bien se ha producido un descenso con respecto al año 2007 (34,9\%) se trata de un porcentaje relativamente elevado que denota cierta debilidad del sistema productivo español para emplear a los jóvenes cualificados y cierta fortaleza del sistema educativo español, ya que una economía como la alemana está demandando jóvenes españoles cualificados para emplearlos en ocupaciones cualificadas. Según los datos ofrecidos por el Observatorio de Bancaja-Ivie, en España hay una generalizada percepción del desajuste subjetivo para el total de los jóvenes y por niveles educativos. En 2011, el 24\% de los jóvenes considera que se encuentra sobrecualificado. Por nivel de estudios, esta proporción aumenta a 
medida que la formación es mayor: el 32\% de los jóvenes con estudios universitarios considera estar sobrecualificado frente al $11 \%$ de aquellos con estudios obligatorios (Bancaja-Ivie, 2012). Este fenómeno tiene consecuencias negativas sobre la productividad de las empresas españolas, su competitividad y el volumen de empleo, suponiendo un claro ejemplo de uso ineficiente de los recursos. El esfuerzo inversor en educación de nuestro país no se transforma en una mayor capacidad productiva en la misma medida que en otros países. En el resto de países esos porcentajes se situaban en 2007 en torno al $21 \%$ o incluso menos, como sucedía en Italia.

Tabla 3: Proporción de jóvenes sobrecualificados $2007-2011$.

\begin{tabular}{lcc}
\hline & $\mathbf{2 0 0 7}$ & $\mathbf{2 0 1 1}$ \\
\hline España & 34,9 & 32,5 \\
Reino Unido & 23,7 & 25,0 \\
Francia & 20,8 & 20,7 \\
Italia & 14,0 & 16,9 \\
Alemania & 21,0 & 21,4 \\
UE 27 & 21,0 & 21,2 \\
\hline
\end{tabular}

Fuente: Elaboración propia a partir de Eurostat, Informe de Juventud, 2013.

Estos datos nos remiten de inmediato a la relación que mantienen educación y empleo en España, atendiendo a la configuración del sistema educativo y a la función que este tiene en la generación de competencias profesionales para favorecer la inserción y empleabilidad de los jóvenes. A este respecto hay que subrayar que la relación entre educación e inserción ocupacional no es un unidimensional sino de una gran complejidad que depende de numerosos factores entre los que se encuentran la globalización, el cambio tecnológico, el envejecimiento de la población y la acusada crisis económica (OECD, 2010; OECD, 2013b; UNESCO, 2012).

Según Ramón García (2011), Dolado et al. (2000) y Moreno Mínguez (2008) las condiciones laborales de la población, en general, y de la juventud, en particular, dependen en gran medida de la eficacia del sistema educativo. En un contexto de exceso de oferta de trabajo y de crecientes requerimientos formativos por parte de las empresas, la probabilidad de que un joven acceda a un empleo estable y adecuadamente remunerado depende, entre otros aspectos, de la cantidad y calidad de la educación recibida. Por tanto, según esta perspectiva las 
causas del desempleo juvenil en España hay que buscarlas en las posibles deficiencias del sistema educativo. En España esta tesis parece parcialmente avalada por la magnitud del abandono escolar temprano y la acusada incidencia del desempleo entre los jóvenes menos formados y los que han abandonado la educación antes de obtener un título de educación secundaria.

El abandono escolar es uno de los indicadores claves para estimar y valorar en qué medida el sistema educativo es eficaz reteniendo a los jóvenes para formarles y favorecer la empleabilidad. El abandono escolar temprano afectaba en el año 2011 al 31,6 \% de los jóvenes españoles, muy superior a la media europea que se sitúa en torno al $15 \%$ para ese mismo año, incluso después de haberse reducido ligeramente en los dos últimos años. Por ejemplo, esta cifra se situaba en torno al $12 \%$ en Alemania y Finlandia, el 16,6\% en Reino Unido y el 14,7\% en Dinamarca según los últimos datos disponibles de Eurostat para 2011. Este cambio de tendencia en España se debe, según los expertos, a los efectos de la crisis económica y al elevado desempleo de los jóvenes; factores que parecen haber puesto en valor la educación (Eurostat, 2009). El abandono escolar tiene un coste importante para la empleabilidad de estos jóvenes. Tal y como se puede apreciar en la figura 2, el desempleo de los jóvenes que han abandonado los estudios prematuramente es relativamente elevado en España.

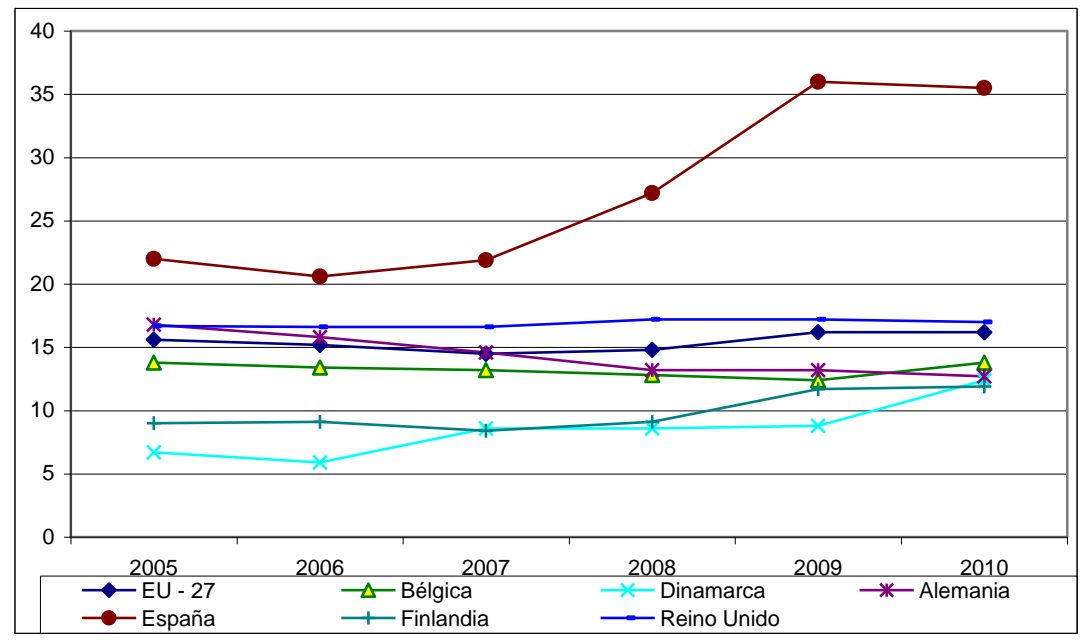

Figura 2. Ratio de desempleo de los jóvenes entre 18 y 24 años que han abandonado el sistema educativo. Fuente: Elaboración propia a partir de Eurostat. 
Este análisis no estaría completo sin incluir datos referidos al desempleo según niveles educativos. Los efectos de la formación sobre el desempleo juvenil y la potencialidad para ser empleado se han hecho evidentes durante la crisis actual, aunque en décadas pasadas ya se dejaban notar sus efectos (Moreno Mínguez, 2008). Los datos de la EPA (Encuesta de Población Activa) evidencian que la crisis ha afectado en mayor medida al empleo de las personas con niveles formativos intermedios-bajos, entre los que se encuentra un elevado porcentaje de jóvenes menores de 19 años con tan sólo educación secundaria de primera etapa (Figura 3).

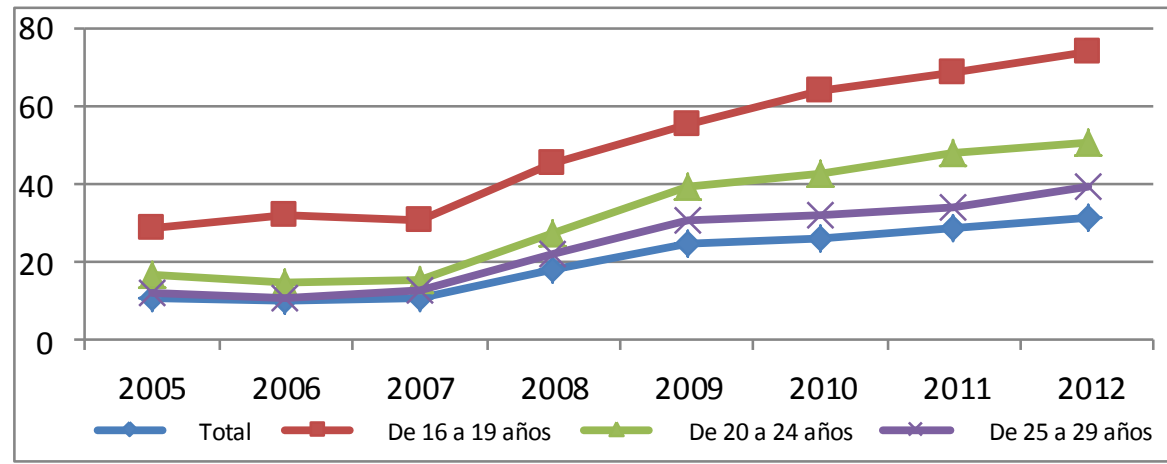

Figura 3. Desempleados con estudios secundarios de primera etapa por edad (\%). Fuente: Elaboración propia a partir de la EPA, INE, Informe de Juventud, 2012.

Los datos también permiten constatar que a mayor edad menor desempleo. Auque el desempleo ha afectado a todos los niveles formativos, en el colectivo de jóvenes con educación universitaria es donde menos empleo se ha destruido en términos comparados, pasando de ser el $7,58 \%$ de desempleados con educación universitaria en 2007 al 23\% en 2012 (Figura 4). Aunque los datos parecen constatar que el desempleo es menor entre aquellos jóvenes con educación secundaria y universitaria, la brecha según nivel formativo se ha incrementado durante la crisis (Felgueroso et al., 2010).

Si bien los datos anteriores han puesto de relevancia que la educación y las fallas del sistema productivo son factores claves para explicar el desempleo de los jóvenes no son menos importantes otros factores relacionados con la clase social, el sexo y la nacionalidad. En las frecuencias por sexo se observa que la tasa de paro es sensiblemente superior entre los varones que entre las mujeres en todos los grupos de edad. Los datos parecen confirmar que las mujeres han resistido mejor a 
los efectos de la crisis en todos los grupos de edad, aunque también es pertinente subrayar que partian de tasas de desempleo más elevadas que los varones (Moreno Minguez, 2013 p. 118).

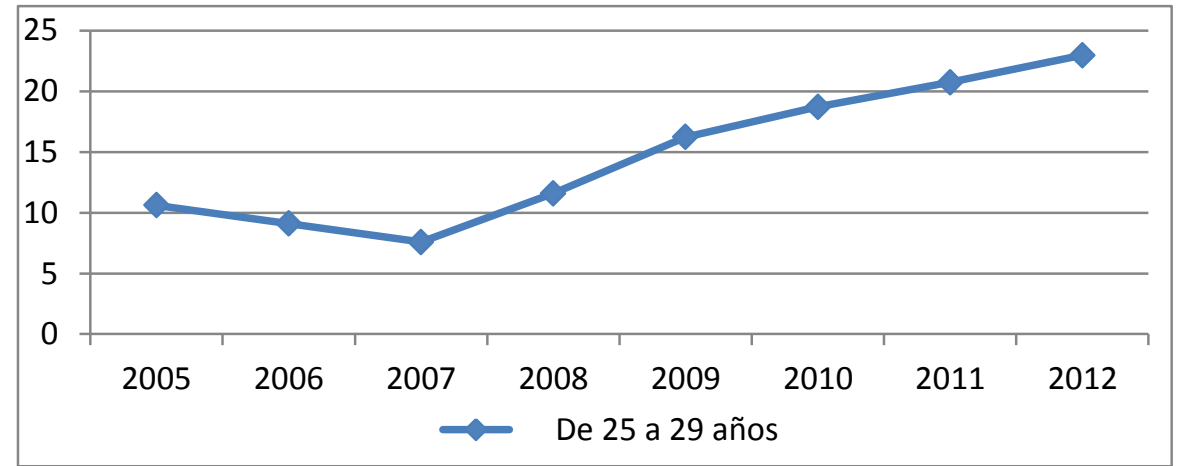

Figura 4. Desempleados con estudios universitarios de 25 a 29 años (\%). Fuente: Elaboración propia a partir de la EPA, INE, Informe de Juventud, 2012.

En lo que se refiere a los jóvenes extranjeros el desempleo ha castigado especialmente al grupo de edad de 16 a 19 (38,4\%). La tasa de paro se ha duplicado para el grupo de edad de 20 a 29 años y se ha triplicado para el colectivo menor de 19 años, que son aquellos que abandonaron la educación sin una cualificación reglada por el sistema educativo (Moreno Mínguez, 2013). Por sexo se observa que el desempleo entre los inmigrantes ha sido mayor entre las mujeres que en el colectivo masculino en todos los grupos de edad, pero especialmente entre las jóvenes menores de 19 años (Figura 5). En la tabla 4, sobre la incidencia diferencial del desempleo entre los jóvenes españoles y extranjeros, se constata que la pérdida de empleo ha sido mayor entre los inmigrantes menores de 25 años que entre los españoles de la misma edad.

En lo que se refiere a la clase social los datos ofrecidos por la Encuesta de Juventud 2012 constatan que a medida que aumenta la clase social disminuye el porcentaje de jóvenes ocupados y se incrementa el de los que sólo estudian (Tabla 5). Esto podría tener la interpretación de que las clases sociales más acomodadas motivan y apoyan a sus hijos para que sólo estudien y se formen adecuadamente para estar mejor preparados para emplearse en el mercado laboral, mientras que las clase sociales más humildes optan en mayor medida por incorporarse tempranamente al mercado laboral. 


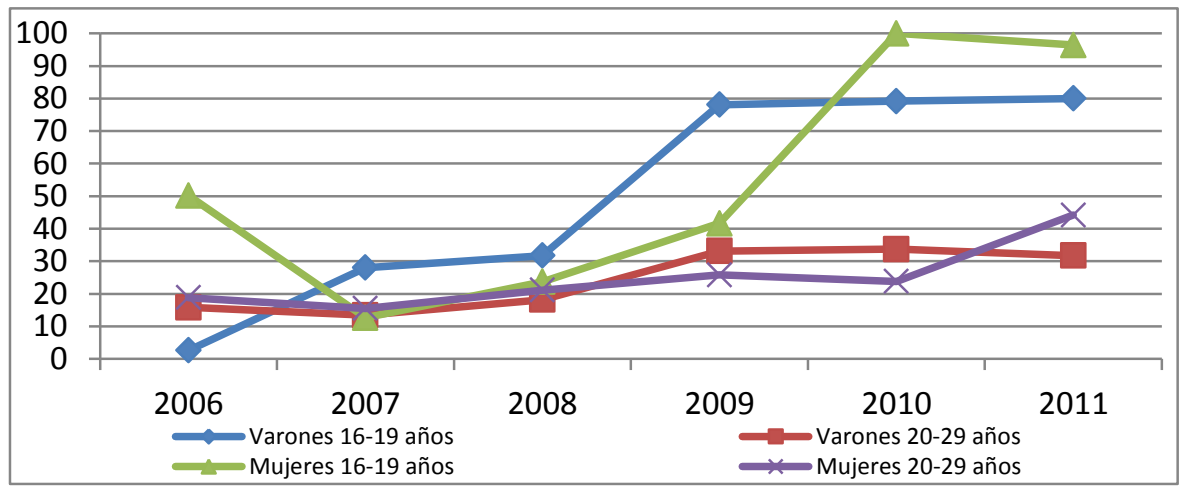

Figura 5. Tasas de paro de los migrantes por sexo y grupos de edad, 20062011. Fuente: Elaboración propia a partir de la EPA, INE, Informe de Juventud, 2012.

Tabla 4. Empleo juvenil (16 a 24 años) en España por nacionalidad 20082012, en miles.

\begin{tabular}{lcccc}
\hline & $\mathbf{2 0 0 8}$ & $\mathbf{2 0 1 2}$ & Diferencia & \% \\
\hline Española & $1.524,30$ & 714,4 & $-809,9$ & $-53,1$ \\
Extranjera & 325,6 & 136,1 & $-189,5$ & $-58,2$ \\
\hline Fuente: Encuesta de Población Activa, en Rocha $(2012)$, Informe de Juventud, 2012.
\end{tabular}

Del mismo modo los datos ofrecidos por los diferentes informes PISA (Programme for International Student Assessment) y que corrobora el último informe de 2013, los empleados jóvenes que ocupan los puestos de trabajo de baja cualificación tienden a encontrarse en los niveles más bajos de rendimiento educativo y de la clase social, lo que aumenta sus probabilidades futuras de estar desempleados. Según estos datos, los jóvenes adultos que han obtenido los mejores resultados en las pruebas PISA son los que, con mayor probabilidad, tienen niveles educativos más altos y han conseguido mejores empleos con más posibilidades de desarrollar sus destrezas (Hernández y Serrano, 2013).

La ambigüedad del concepto de empleabilidad y la falta de consenso sobre las causas del elevado desempleo juvenil se refleja en la escasa efectividad que han tenido las políticas laborales y educativas diseñadas para paliar el desempleo juvenil en España (Betcherman, Godfrey, Puerto, Rother y Stavreska 2007). 
Tabla 5. Jóvenes (\%) según relación con la actividad por clase social, 2012. $(\mathrm{N}=4835)$

\section{Clase social}

\begin{tabular}{lccccc} 
& Alta & Media alta & Media media Media baja & Baja \\
\hline Sólo trabajo & 15,1 & 22,6 & 20,4 & 22,8 & 16,5 \\
Principalmente trabajo y además estudio & 10,4 & 10,2 & 7,5 & 6,0 & 7,9 \\
Principalmente estudio y hago algún trabajo & 8,3 & 15,5 & 12,8 & 9,1 & 12,8 \\
Sólo estudio & 47,8 & 32,6 & 36,7 & 38,2 & 34,7 \\
Estudio y además estoy buscando trabajo & 11,4 & 8,7 & 7,7 & 8,5 & 7,9 \\
Estoy buscando mi primer trabajo & 2,0 & 1,7 & 1,9 & 2,2 & 1,8 \\
Estoy en paro cobrando desempleo & 0,7 & 2,0 & 2,8 & 4,1 & 3,7 \\
Estoy en paro sin cobrar desempleo & 3,3 & 5,4 & 8,5 & 6,8 & 11,0 \\
Otra situación & 1,0 & 1,3 & 1,7 & 2,3 & 3,7 \\
\hline Total & $100 \%$ & $100 \%$ & $100 \%$ & $100 \%$ & $100 \%$ \\
\hline
\end{tabular}

Fuente: INJUVE, elaboración propia a partir de la Encuesta de Juventud, IJE 2012.

Las razones de este hecho están en que la legislación laboral en España ha favorecido la dualidad y segmentación del mercado de trabajo fomentado la temporalidad en el empleo. El origen de la temporalidad se remonta a mediados de los ochenta cuando, para combatir el fuerte incremento de la tasa de paro a consecuencia del efecto retardado de las crisis del petróleo y el proceso de reindustrialización durante la transición democrática, en 1984 se aprobó una reforma radical del mercado de trabajo. Dicha reforma permitió el uso indiscriminado de la contratación temporal (con reducidos costes de despido) para cualquier actividad productiva regular, al tiempo que mantenía inalterada la rígida protección de los contratos indefinidos a través de elevadas indemnizaciones por despido (Dolado, García y Jimeno, 2002).

De acuerdo con Cabrales et al., (2013) la tasa de temporalidad (es decir, la proporción que representan los trabajadores con contrato temporal sobre el total de asalariados) se disparó desde un 15\% antes de la reforma de 1984 hasta el 35,4\% a mediados de los noventa. Desde entonces, un porcentaje superior al 90\% (el 94\% en la actualidad) de las nuevas contrataciones se han producido en esta modalidad contractual, mientras que la tasa media de conversión de contratos temporales a indefinidos ha oscilado entre el $10 \%$ en los noventa y primera mitad de los 2000 , para pasar a situarse en el 5\% en la actualidad (González y Miles, 2012; Güell y Petrongolo, 2007). Posteriormente, tras una larga sucesión de reformas laborales parciales, la tasa de temporalidad se estabilizó alrededor de un 30\%. Tras la 
masiva destrucción de empleos temporales durante la recesión que lleva afectando a España desde 2008, dicha tasa solo se ha reducido hasta el 23\%, manteniéndose todavía como una de las más altas en la OECD. En la figura 6, se puede comprobar como la temporalidad es una constante en el empleo de los jóvenes desde el año 2000, muy superior a la media europea. Esto podría ser un factor explicativo más de cómo la destrucción de empleo en las ocupaciones temporales, concentradas fundamentalmente en la población juvenil y constado por numerosos informes (OECD, 2010; OECD, 2013a; Moreno Minguez, 2013), ha afectado especialmente a los jóvenes con tasas de temporalidad cercanas al ochenta por ciento y por tanto ha contribuido a incrementar el desempleo juvenil.

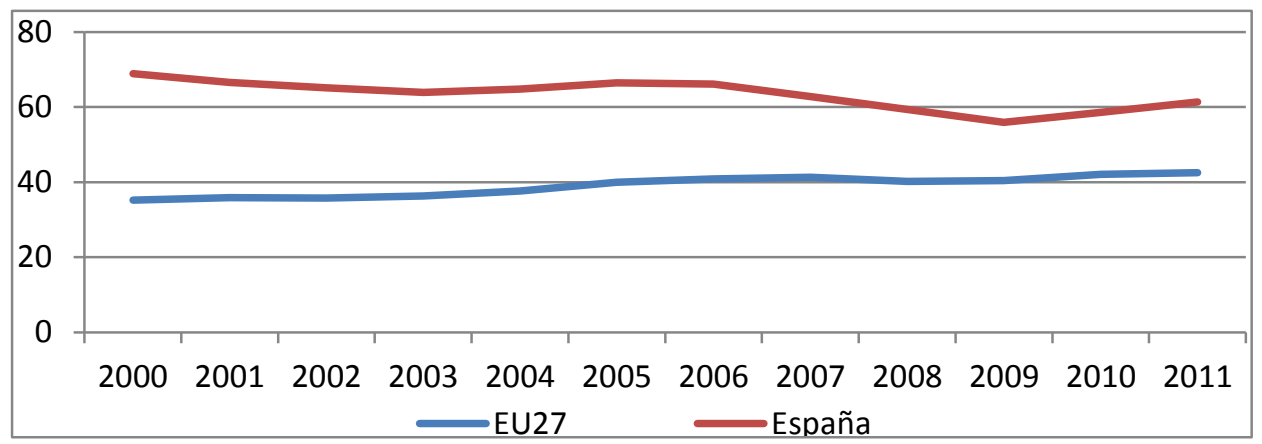

Figura 6. Trabajadores temporales de 15 a 24 años del total de jóvenes de la misma edad (\%) por sexo 2000-2011. Fuente: Elaboración propia a partir de Eurostat, Informe de Juventud, 2012.

\section{CONCLUSIONES}

En este artículo se ha reflexionado sobre el ambiguo concepto de empleabilidad y su aplicabilidad como instrumento teórico para interpretar el desempleo juvenil en España en un contexto de crisis. Generalmente se asocia este concepto con el capital formativo del trabajador, sin tener en cuenta factores tales como el sexo, la nacionalidad, la clase social de pertenencia o las propias deficiencias del sistema productivo y formativo. Los datos aportados en este trabajo destacan la falta de consenso entre los investigadores sobre los factores determinantes de la reducida empleabilidad de los jóvenes españoles. Los desajustes existentes entre la formación recibida y las necesidades del mercado laboral, así como los fallos del propio sistema productivo se apuntan como factores intervinientes claves en la explicación del desempleo juvenil, aunque estos resultados no son concluyentes. Por 
un lado, el desajuste existente entre la formación recibida y la empleabilidad de los jóvenes se refleja claramente en el elevado desempleo de los titulados superiores y en el abandono escolar prematuro como ejemplos de los problemas que tiene el sistema educativo español para formar adecuadamente e integrar a los jóvenes en el mercado laboral desde el inicio de la crisis económica. Por otra parte, los problemas del sistema productivo para emplear a los jóvenes se reflejan en las características de los empleos que se han creado y destruido entre el colectivo de los jóvenes desde el inicio de la crisis, así como en la evolución que ha tenido la sobrecualificación. Los datos aportados en este artículo constatan que se han destruido fundamentalmente empleos temporales, ocupados en un $80 \%$ por jóvenes, empleos intermedios y descualificados, además de subrayar que la sobrecualificación de los jóvenes que están trabajando es una de las más elevadas de Europa.

Otra conclusión relevante de este estudio a partir de los datos aportados es que el desempleo no se distribuye de forma homogénea entres todos los jóvenes, ya que son los de menor edad, aquellos con menor formación, los inmigrantes y los jóvenes situados en los estratos más bajos de la estructura social los que tienen mayores tasas de desempleo.

En definitiva, el elevado desempleo juvenil existente en España ha puesto de manifiesto las limitaciones operativas de las políticas de empleo basadas en el concepto de empleabilidad para activar la inserción laboral de los jóvenes en un contexto de crisis económica. Los resultados aportados en este artículo deberian servir de referencia para orientar las políticas públicas en base a la necesidad de reformular el sistema productivo español invirtiendo en $\mathrm{I}+\mathrm{D}+\mathrm{I}$, creando puestos de trabajo cualificados e invirtiendo en políticas públicas de educación que frenen el abandono escolar prematuro y fomenten la formación continua entre los jóvenes con escasa formación. Por otra parte también se hace necesario intervenir en aquellos colectivos de jóvenes con mayores dificultades para emplearse como son los de menor edad, los que pertenecen a las clases sociales menos acomodadas y los inmigrantes, que además tienen un elevado riesgo de sufrir exclusión social. Sólo así se podrá hacer frente al desempleo juvenil como factor clave de cohesión social y de igualdad social en una sociedad severamente castigada por los efectos de la crisis económica.

\section{REFERENCIAS}

Banco de España. (2013). Evolución del desajuste educativo entre la oferta y la demanda de trabajo en España. Boletín económico, $\mathrm{n}^{\circ} 50$.

Bancaja-IVIE. (2011). Crisis económica e inserción laboral de los jóvenes. Valencia: Bancaja- IVIE. 
Bancaja-IVIE. (2012). Datos del observatorio de inserción laboral de los jóvenes. Valencia: IVIE.

Beck, U., y Beck-Gernsheim, E. (2002). Individualization: Institutionalized individualism and its social and political consequences. London: Sage.

Bentolila, S., Cahuc, P., Dolado, J., y Le Barbanchon, T. (2012). Two-Tier Labour Markets in a Deep Recession: France vs. Spain. The Economic Journal, 122, 155-187.

Betcherman, G., Godfrey, M., Puerto, S., Rother, F., y Stavreska, A. (2007). A review of interventions to support young workers: Findings of the youth employment inventory. World Bank Social Protection Discussion Paper, 715, (Washington D.C.).

Blázquez, M. (2005). Youth labour market integration in Spain: Search time job duration and skill mismatch. Spanish Economic Review, 7, 191-208.

Breen, R., y Goldthorpe, J. (1999). Class, mobility, and merit: The experience of two British cohorts. European Sociological Review, 17, 81-101.

Cabrales, A., Dolado, J., y Mora, R. (2013). Dualidad laboral y déficit de formación en el empleo: evidencia sobre España con datos PIAAC. En Instituto Nacional de Evaluación Educativa (Eds.), PIAAC: Programa Internacional para la Evaluación de las Competencias de la población adulta, 2013, (Informe español vol. II).

Council of the European Union. (2010). European cooperation in the youth field (2010-2018). Estrasburgo.

Dolado, J., Felgueroso, F., y Jimeno, J. (2000). Youth labour markets in Spain: Education, training, and crowding-out. European Economic Review, 44 (4-6), 943-956.

Dolado, J., García, C., y Jimeno, J. (2002). Drawing lessons from the boom of temporary jobs in Spain. The Economic Journal, 11 (721), 270-295.

Dolado, J., Jansen, M., Felgueroso, F., Fuentes, A., y Wölfl, A. (2013). Youth labour market performance in Spain and its determinants: A Micro-Level Perspective, OECD. Economics Department Working Papers $n^{\circ} 1039$, (Paris: OECD Publishing).

Erikson, R., y Jonsson, J. (1996). Explaining class inequality in education: The Swedish case in comparative perspective. Oxford: Westview Press.

European Commission. (2012). Joint Report of the Council and the Commission on the implementation of the renewed framework for European cooperation in the youth field (2010-18) (2012/C 394/03).

European Commission. (2013). Youth employment: Commission welcomes Council agreement on youth guarantee. Memo/13/152.

European Union. (2013). ESCO, 2020, European Classification of Skills/Competences, Qualifications and Occupations. Luxembourg: Publications Office of the European Union.

Eurostat. (2009). Youth in Europe: A statistical portrait, Luxembourg: Publications Office of the European Union.

Garrido, L. (2012). Para un diagnóstico sobre la formación y el empleo de los jóvenes. Cuadernos, 2, (Empleo Juvenil, Círculo Cívico de Opinión).

Felgueroso, F. (2012). El empleo juvenil en España: Un problema estructural. Círculo Cívico de Opinión.

Felgueroso, F., Hidalgo, M., y Jiménez-Martín, S. (2010). Explaining the fall of the skill wage premium in Spain. En Cabrales, A. y Celentani, M. (coordinadores), Talent, effort and social mobility (p. 175-230). Madrid: Colección Monografias Fedea. 
González, X., y Miles, D. (2012). Labor market rigidities and economic efficiency: Evidence from Spai. Labour Economics, 20 (6), 833-45.

Güell, M., y Petrongolo, B. (2007). How binding are legal limits? Transitions from temporary to permanent work in Spain. Labour Economics, 14, 153-183.

Hernández, L., y Serrano, L. (2013). Efectos económicos de la educación en España. En Instituto Nacional de Evaluación Educativa (Eds.), PIAAC: Programa Internacional para la Evaluación de las Competencias de la población adulta, 2013, (Informe español vol. II).

Instituto de la Juventud de España. (2011). Desmontando a los nini: Un estereotipo juvenil en tiempos de crisis. Madrid: Instituto de la Juventud de España.

Martínez, J. S. (2013). Sobrecualificación de los titulados universitarios y movilidad social. En Instituto Nacional de Evaluación Educativa (Eds.), PIAAC: Programa Internacional para la Evaluación de las Competencias de la población adulta, 2013, (Informe español vol. II).

Moreno Mínguez, A. (2008). Economía, Empleo y consumo de los jóvenes (Tomo II), Informe de Juventud. Madrid: Ministerio de Sanidad, Politica Social e Igualdad.

Moreno Minguez, A. (2012). The late transition to adulthood in Spain in a comparative perspective: The incidence of structural factors. Young: Nordic Journal of Youth Research, 20(1), 19-48.

Moreno Mínguez, A. (2013). Informe de Juventud 2012. Madrid: Instituto de la Juventud de España.

OECD. (2010). Off to a good start? Jobs for youth. Paris: OECD Publishing.

OECD. (2013a). Education at a Glance 2013. Paris: OECD Publishing.

OECD. (2013b). OECD skills outlook 2013 first results from the survey of adult skills. Paris: OECD Publishing.

OIT. (2013). Youth guarantees: A response to the youth employment crisis? Geneva: Employment Policy.

Pérez, F., y Hernández, L. (2013). Educación, conocimientos y perfiles ocupacionales. En Instituto Nacional de Evaluación Educativa (Eds.), PIAAC: Programa Internacional para la Evaluación de las Competencias de la población adulta, 2013, (Informe español vol. II).

Ramón García, J. (2011). Desempleo juvenil en España: Causas y soluciones. BBVA Research, 11/30 Documentos de Trabajo.

Rodríguez Cuba, J. (2009). Empleabilidad de los jóvenes. Serie Avances de Investigación, 32. Madrid: Fundación Carolina.

Rocha, F. (2012). El desempleo juvenil en España: Situaciones y recomendaciones politicas. Madrid: Fundación $1^{\circ}$ de Mayo, CC.OO.

Schoon, I. (2008). A trasgenerational modelo of status attainment: The potential mediating role of school motivation and education. National Institute Economic Review, 205, 72-82.

Schoon, I., y Silbereisen, R. (Eds.) (2009). Transitions from School to Work: Globalisation, Individualisation, and Patterns of Diversity. New York: Cambridge University Press.

Shanahan, M. (2000). Pathways to adulthood in changing societies: Variability and mechanisms in life course perspective. Annual Review of Sociology, 26, 667-692.

Pissarides, Ch. (2011). The Future of Work in Europe. CentrePiece-The Magazine for Economic Performance, 354, (Centre for Economic Performance, LSE).

UNESCO. (2012). Youth and skills, putting education to work, 2012 education for all monitoring report. Paris: UNESCO. 